\title{
G

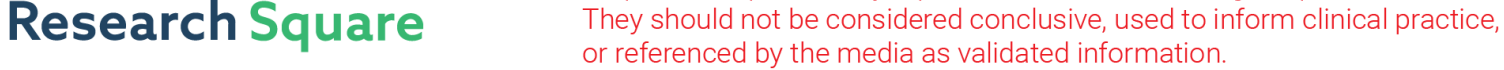 \\ LncRNA APOC1P1-3 Promoting Anoikis-resistance of Breast Cancer Cells
}

\section{Qi Lu}

Fudan University School of Basic Medical Sciences

\section{Li Wang}

Fudan University School of Basic Medical Sciences

\section{Ping Zhu}

Fudan University School of Basic Medical Sciences

\section{Yabiao Gao}

Fudan University School of Basic Medical Sciences https://orcid.org/0000-0003-2014-6050

\section{Luying Li}

Fudan University School of Basic Medical Sciences

\section{Xue Wang}

Fudan University School of Basic Medical Sciences

\section{Youping Jin}

Fudan University School of Basic Medical Sciences

\section{Xiuling Zhi}

Fudan University School of Basic Medical Sciences

Jerry Yu

Louisville Medical Institute: University of Louisville

\section{Xin Li}

Shanghai 9th Peoples Hospital Affiliated to Shanghai Jiaotong University School of Medicine

\section{Xingjun Qin}

Shanghai 9th Peoples Hospital Department of Ophthalmology

\section{Ping Zhou ( $\nabla$ zping@shmu.edu.cn )}

Department of Physiology and Pathophysiology, School of Basic Medical Sciences, Fudan University, No. 130 Dongan Road, Shanghai, 200032, China; https://orcid.org/0000-0002-4183-0639

\section{Primary research}

Keywords: breast cancer, IncRNA APOC1P1-3, anoikis resistance, miRNA-188-3p, tumor metastasis

Posted Date: December 15th, 2020

DOl: https://doi.org/10.21203/rs.3.rs-125353/v1 
License: (c) (i) This work is licensed under a Creative Commons Attribution 4.0 International License. Read Full License

Version of Record: A version of this preprint was published at Cancer Cell International on April 26th, 2021. See the published version at https://doi.org/10.1186/s12935-021-01916-w. 


\section{Abstract}

Background: Anoikis resistance plays a critical role in the tumor metastasis by allowing survival of cancer cells in the systemic circulation. We previously showed that long non-coding RNAs APOC1P1-3 (IncRNA APOC1P1-3) inhibits breast cancer cell apoptosis. However, its role in the anoikis resistance remains unclear.

Methods: We induced anoikis resistance in two breast cancer cell lines (MCF-7 and MDA-MB-231) under anchorage-independent culture condition and studied the effects of IncRNA APOC1P1-3 on the apoptosis. The Dual-Luciferase activity assay were used to whether miRNA-188-3P can specifically bind to IncRNA APOC1P1-3. We further explored the role of APOC1P1-3 in the lung metastasis by injecting MDA-MB-231 and MDA-MB-231-APOC1P1-3-knock-down cells in the female BALB/c nude mice.

Results: We found that it suppressed early apoptosis of these cells by gain or loss of their function, respectively. We further explored its mechanism related to anoikis resistance, and found this molecule promoted the resistance via activating Caspase 3, 8, 9 and PARP. Moreover, it was specifically binding to the target miRNA-188-3p to block its inhibition of Bcl-2 (an anti-apoptosis protein). These findings suggest that IncRNA APOC1P1-3 plays an important role in the development of breast cancer metastasis via anoikis resistance.

Conclusions: This study demonstrates that IncRNA AP0C1P1-3 can promote the anoikis resistance of breast cancer cells and specifically bind to miRNA-188-3p acting as a "sponge" to block the inhibition of anti-apoptotic protein Bcl-2.

\section{Introduction}

Metastasis, the major cause of death in cancer patients, is a complicated process involving detachment of cancer cells from the original site, survival in the circulatory and lymphatic systems, and colonization in distant target organs [1]. Anoikis resistance is a prerequisite for metastasis, facilitating secondary tumor formation in distal organs [2]. Anoikis is a particular type of apoptosis of epithelial and endothelial cells as losing attachment from the appropriate extracellular matrix (ECM) or neighboring cells. It is a physiological process for organ development and tissue homeostasis [3]. Anoikis resistance plays a vital role in the tumor survival via circulating tumor cells in the blood [4]. It involves in many intracellular molecules, such as growth factor receptors, microRNAs (miRNAs) and long noncoding RNAs (IncRNAs) [5].

LncRNAs are a set of key regulatory RNAs with transcripts longer than 200 nucleotides. Increasing evidence indicates that they involve in almost all physiological and pathological processes in the human body, including cell proliferation, apoptosis, stem cell maintenance, and cancer metastasis $[6,7,8,9]$. They directly regulate the metastasis both in vitro and in vivo. LncRNA HOTAIR aberrantly expressed in many tumors (liver, breast, colon and pancreatic cancers), contributing to the metastasis [10, 11]. LncRNA MALAT1 was discovered first in non-small cell lung cancer patients with high metastasis and poor 
prognosis [12]. It was upregulated in prostate, colorectal, and bladder cancers [13, 14, 15]. IncRNAs were also involved in various mechanisms, including sponge of miRNA, epithelial-mesenchymal transition (EMT) and epigenetic gene regulations [16]. However, their mechanisms in the anoikis resistance of breast cancer remains unclear. In our previous studies, we sought out long intergenic non-coding RNA APOC1P1-3 from breast cancer tissues and cell lines, and found that it was upregulated in most cases, and correlated with tumor size and hypomethylation in its promoter region. Moreover, it promoted cell proliferation and inhibited apoptosis via a-tubulin acetylation [17]. In the present study, we further explored its role in the anoikis resistance of breast cancer.

\section{Methods}

\subsection{Human breast cancer tissues and cell lines}

A total of 24 freshly resected breast cancer samples and paired paracancerous tissues were collected from Fudan University Shanghai Cancer Center. Our present research has been approved by Basic Medical Ethics Committee of the Fudan University (2017-F001). The patient signed the informed consent. Specimens were snap-frozen in liquid nitrogen immediately after resection and kept in $-80^{\circ} \mathrm{C}$.

The breast cancer MCF-7 and MDA-MB-231 cell lines were obtained from the cell bank of the Shanghai Institute of Biological Sciences, Chinese Academy of Sciences. The cells were cultured in DMEM/F12 medium with $10 \%(\mathrm{v} / \mathrm{v}$ ) fetal bovine serum (Gibco, USA), 100 units penicillin and $100 \mu \mathrm{g} / \mathrm{ml}$ streptomycin, at $37^{\circ} \mathrm{C}$ with $5 \% \mathrm{CO}_{2}$.

\subsection{Cytoplasmic and nuclear separation}

The content of RNA in cytoplasm and nucleus was studied with PARISTM Kit (Thermofisher AM1921). When the confluence rate was $>90 \%$ in the $60 \mathrm{~mm}$ dish, the cells were collected. Cytoplasm and nuclear were separated by centrifugation following the manual of kit. The cytoplasm supernatant and nucleus precipitate were transferred to different tubes, equal volume of $2 \bigotimes$ Lysis Binding Buffer and mixed thoroughly with a pipette. The obtained RNA was stored at $-80^{\circ} \mathrm{C}$ with an appropriate amount of DNAase to prevent degradation.

\subsection{RNA-Chip and bioinformatics analysis}

The expression of IncRNA was screened by pairwise random variance in the cancer and control tissue samples. The specific criteria were: IncRNA expression was 1.5 times higher in cancer tissue, and the $\mathrm{P} \leq$ 0.05. The relevant hierarchical clustering analysis was assisted by Kang Cheng Biotechnology Co, Ltd., using Cluster Treeview software (Standford).

\subsection{Real-time quantitative PCR}

RT-PCR was used to verify the expression of IncRNA APOC1P1-3. Total RNA from cells and tissues was extracted by TRIzol from Invitrogen. Reverse transcription was performed with TOYOBO's ReverTra Ace 
qPCR RT Kit. Real-time PCR was performed with Bio-RAD iTaqTM Universal SYBRß Green Supermix. The primer sequences are described in Table S4.

\subsection{Anoikis model of breast cancer cells}

Anoikis model was established by poly-2-hydroxycthyl methacrylate (polyHEMA) (Sigma company) culture [35]. PolyHEMA was dissolved in ethanol (final concentration of $120 \mathrm{mg} / \mathrm{ml}$ ) in a $65^{\circ} \mathrm{C}$ water bath. The PolyHEMA solution (1:10 in 95\% ethanol) was sterilized under a $0.22 \mu \mathrm{m}$ filter and pipetted into a 6-well culture plate $(1 \mathrm{ml} /$ well). The plate was incubated overnight at room temperature, washed three times with PBS, and incubated with DMEM/F12 medium prior to use. $1 \times 10^{6}$ cells were seeded into the PolyHEMA-coated wells for $12,24,48$ or $72 \mathrm{~h}$ in a humidified $\left(37^{\circ} \mathrm{C}, 5 \% \mathrm{CO}_{2}\right)$ incubator.

\subsection{Annexin V-FITC/PI double staining for apoptosis}

MDA-MB-231 a and MCF-7a cells cultured for $48 \mathrm{~h}$ were collected, digested with 0.25\% EDTA-free trypsin and pipetted to achieve a uniform single cell suspension. Cell concentration was adjusted to L-15/DMEM medium containing 10\% FBS, $10 \%$ double antibiotics to $1 \times 10^{6}$ cells $/ \mathrm{ml}$. The Annexin V-FITC/PI double staining was performed with the Annexin V $\mathrm{FFITC}$ Apoptosis Detection Kit (Dojindo).

\subsection{Dual-Luciferase report gene vectors}

The pmirGLO Dual-Luciferase miRNA Target Expression Vector and Dual-Luciferase ${ }^{\circledR}$ Reporter Assay were purchased from Promega. Using bioinformatics to analyze and predict the binding site of miRNA188-3p to AP0C1P1-3, we designed primers and cloned the target fragment. The fragment was inserted into the pmirGLO Dual-Luciferase miRNA Target Expression Vector by molecular cloning, and the recombinant reporter plasmid and the target microRNA mimic were co-transfected into the target cells. The luciferase was extracted and its activity was tested. The mutated fragment was ligated into the dual luciferase reporter gene vector, and the target microRNA mimetic was co-transfected with the target cell for fluorescence detection. The DNA purification kit Glue recovery kit and Rapid site-directed mutagenesis kit were purchased from TIANGEN. Endonuclease XhollSall and T4 ligase were from NBE.

\subsection{In vivo metastasis studies}

We firstly designed a lentivirus for knock-down human APOC1P1-3 (5' GAAAGACCCTGGAGGACTA 3') from GeneChem Corporation (Shanghai, China). We seeded $2 \times 10^{5}$ MBA-MD-231 cells in 6-well plates and carried out the transfection with Lv-sh-APOC1P1-3 and Lv-sh-Control $24 \mathrm{~h}$. At $72 \mathrm{~h}$, we extracted total RNA from the cells and evaluated the inhibitory effects (about 60\%) with RT-PCR (Supplementary materials). Then, we established the AP0C1P1-3 knock-down and control stable MDA-MB-231 cell lines with transfected Lv-sh-APOC1P1-3, Lv-sh-Control and the Puromycin $(2.5 \mu \mathrm{g} / \mathrm{ml})$.

All animal experiments were performed in accordance with the guidelines and approved by the Institutional Animal Care and Use Committee at Fudan University (20160225-108). Six-week-old female BALB/c nude mice were used (8 mice/group). MDA-MB-231 and MDA-MB-231 with AP0C1P1-3 knock- 
down cells $\left(2 \times 10^{6}\right.$ cells/group) were injected into the tail vein. After 7 weeks, the mice were sacrificed and lung metastases were quantified and imaged from Hematoxylin and Eosin (HE) stained slides.

\subsection{Statistical analyses}

All values are reported as mean \pm SE and analyzed with the SPSS 17.0 (Chicago, IL, USA). For comparisons, one-way analyses of variance, Fisher's exact tests, $\mathrm{X}^{2}$-tests, and two-tailed student's t-tests were performed. $P \leq 0.05$ was considered to be statistically significant. The diagrams were completed with Prism 5.0 (GraphPad Software, La Jolla, CA, USA).

\section{Results}

\subsection{Characterization of IncRNA APOC1P1-3}

Previously, we measured and compared IncRNA APOC1P1-3 expression in breast cancer and its adjacent tissues via microarray chip. In order to identify its protein-noncoding property, we checked the Open Reading Frame (ORF) of APOC1P1-3 by ORF finder (http://www.ncbi.nlm.nih.gov/orffinder/), and found that it has 8 ORFs and none is more than 200nt, suggesting no protein coding potential (Table S1). CSF is the mutation rate of the codon. Codons are relatively conservative in the coding region, but prone to mutations in the non-coding region. Due to the problem of sequence conservation, Michael [18] proposed a new value to introduce the evolution model PhyloCSF. We also checked the PhyloCSF of APOC1P1-3 on UCSC (http://genome.ucsc.edu/). All values in the exon of APOC1P1-3 are less than 0, indicating no conservative type.

\subsection{Correlation between IncRNA-APOC1P1-3 and breast cancer}

We measured the expression of IncRNA AP0C1P1-3 in 3 malignant (T47D, MCF-7, MDA-MB-231) and a normal (MCF-10A) breast cell lines and in breast cancer tissues, by real-time qPCR (Figure. 1a,1c). The expressions in malignant cells upregulated, especially in the MDA-MB-231 cells (increased by 1.5 fold). In addition, IncRNA AP0C1P1-3 was found both in cytoplasm and nucleus in MDA-MB-231 cells (Figure. 1b). To evaluate the role of IncRNA APOC1P1-3 in survival, we used the cBioPortal database in TCGA for related bioinformatics analysis. The survival rate is much higher in patients with low expression (blue line) than with high expression (red line) (Figure. $1 d ; n=816, P<0.01$ ). This may be related to distant metastasis of the tumor. We further explored the role of APOC1P1-3 in the lung metastasis by injecting MDA-MB-231 and MDA-MB-231-APOC1P1-3-knock-down cells into the tail vein, and found that the lung metastases burden in nude mice of APOC1P1-3-knock-down cells significantly decreased (Figure. $1 \mathrm{e}(\mathrm{i}), \mathrm{e}(\mathrm{ii}))$.

\subsection{The capacity of anoikis resistance of breast cancer cells}

Anoikis resistance model was established by culturing the cells on a low-adherent culture plate with polyHEMA [19]. Both MDA-MB-231 and MCF-7 cells survived under adherent and anchorage-independent 
conditions for $72 \mathrm{~h}$ by forming micro-tissues (Figure. 2a,2b), indicating the anoikis resistance. MCF-7a aggregated in large clusters with more cells were dead than MDA-MB-231a, which is similar to a previous report [20]. MDA-MB-231a cells formed many small irregular clumps, which may be responsible for detachment from primary tumor tissues.

Under both adherent culture and ultralow attachment culture, we collected cells with or without underwent anoikis resistance for 48 h. Aggregation occurred in all cells (MCF-7a, Figure. 2c and MDA-MB-231a, Figure. $2 d)$. The aggregation was much more in the anoikis group than in the normal group $(n=7, * \star P<$ $0.01)$.

We compared the number of apoptotic cells under adherent culture and low-adherent culture condition Fluorescence-activated cell sorting (FACS). After $48 \mathrm{~h}$ culture, the numbers of apoptotic cells were significantly fewer in MCF-7a and MDA-MB-231a (Figure. 2e-f) than in MCF-7 and MDA-MB-231 groups $(n=3, * * P<0.01)$, indicating an effective induction of anoikis resistance.

\subsection{LncRNA APOC1P1-3 suppressing apoptosis via apoptosis-related proteins}

In the gain/loss functionality studies, the expression of IncRNA APOC1P1-3 efficiently up-regulated by transfection with pcDNA3.1-sense in MCF-7 cells and down-regulated by transfection with siRNA-196 in MDA-MB-231 cells (Figure. 3a-b). The percentage of early apoptosis (FACS) was significantly decreased in MCF-7a cells (Figure. 3c) $(n=3, * P<0.05)$, and increased in MDA-MB-231a cells (Figure. 3d) $(n=3, * P<$ 0.05). The results support that the IncRNA APOC1P1-3 could promote anoikis resistance.

To probe the mechanisms of anoikis resistance, we measured apoptosis related proteins (Western Blot) from MCF-7a transfected with siRNA-196, as well as MDA-MB-231a transfected with pcDNA3.1-sense (Figure. 3e-f) $(n=3, \star \star P<0.0)$. The expressions of activated-caspase $3,8,9$, and activated-PARP upregulated in MDA-MB-231a cells which were knockdown with siRNA-196. In contrast, the expressions decreased in MCF-7a cells which were overexpressed with pcDNA3.1-sense. These results indicate that upregulation of IncRNA APOC1P1-3 could influence anoikis resistance via caspase-dependent pathway.

\subsection{Regulation of anoikis resistance via binding to miRNA- 188-3p}

We used RNA microarrays (provided by Kangcheng Company) to screen related target microRNAs of IncRNA-APOC1P1-3 in breast cancer tissues, and predicted microRNAs in multiple databases like PITA, MIRDB4.0 and Miranda by matching between sequences (Table. S2). There are 84 microRNAs that may bind to APOC1P1-3. With Venn diagram to compare the predicted results of databases and chip, miRNA188-3p most likely binds to IncRNA APOC1P1-3 (Figure. 4a). The microarray results suggest that the binding site is at 222 bp-248 bp of APOC1P1-3 (Figure. 4b).

The pmirGLO-APOC1P1-3'UTR and the pmirGLO-APOC1P1-Mut vectors were separately constructed with the Dual-Luciferase Report Gene System. We used the BiBiServ-RNAhybrid database to perform 
thermodynamic analysis of the predicted binding sites before making a point mutation. The mfe value of the predicted site is $-28.5 \mathrm{kcal} / \mathrm{mol}$ (Figure. S1), the highest absolute value among all 11 predicted sites, indicating a good thermodynamic effect. Group $4 \mathrm{mfe}$ random mutation was $-25.4 \mathrm{kcal} / \mathrm{mol}$ (Table. S3), indicating a better thermodynamic effect.

By transfected miRNA 188-3p mimics, we found that the expression of miRNA-188-3P was significantly higher than that in the control group (Figure. 4c). The pmirGLO-APOC1P1-3'UTR plasmid was bound to the co-transfected miRNA-188-3P mimics. Since the transcription of the luciferase reporter gene was blocked, the fluorescence activity was significantly lowered. After mutation (Figure. S2), miRNA-188-3P mimics could no longer bind to it, and the luciferase reporter gene was transcribed normally (Figure. 4d). The fluorescence activity was not significantly different from the control, suggesting that miRNA-188-3P can specifically bind to IncRNA-APOC1P1-3.

\subsection{The cross-talking between miRNA-188-3p and IncRNA- AP0C1P1-3}

We further explored the effect of miRNA-188-3p on anoikis resistance. miRNA-188-3p was first overexpressed in MDA-MB-231a cells by transfection with miRNA-188-3p mimics. Early apoptosis was highly increased after the overexpression (Figure. 4e), suggesting an inhibition of anoikis resistance. After co-overexpression of IncRNA APC1P1-3 and miRNA-188-3p, there was no significant difference in early apoptosis between Sense + Mimics and the controls (Figure. 4f, revealing that miRNA-188-3p can reverse the effect of IncRNA-APOC1P1-3 in anoikis resistance.

Moreover, we studied the mutual regulation between IncRNA APOC1P1-3 and miRNA-188-3p. Firstly, we overexpressed APOC1P1-3 in MCF-7a cells by transfection with pCDNA3.1-sense, and evaluated the expression of miRNA-188-3p, and vice versa. Neither overexpression of miRNA-188-3p nor IncRNAAPOC1P1-3 caused a change (Figure. 4g-h). Thus, although IncRNA APOC1P1-3 binds to miRNA-188-3p, the two molecules do not have any mutual impact.

\subsection{LncRNA-AP0C1P1-3 synergizes with miRNA-188-3p to affect Bcl-2}

To study the effect of IncRNA APOC1P1-3 and miRNA-188-3p on the anti-apoptotic protein Bcl-2, we examined the Bcl-2 expression in 4 groups (sense+/mimics-, sense-/mimics+, sense+/mimics+, sense-/mimics-) (Figure. 5a-c). Overexpression of IncRNA APC1P1-3 (sense+/mimics-), significantly increased Bcl-2 ( $n=3, * \star P<0.01)$, while overexpression of miRNA-188-3p (sense-/mimics + ) decreased it $(n=3, * * P<0.01)$. Interestingly, overexpression of both IncRNA APOC1P1-3 and miRNA-188-3p (sense + /mimics + ) did not alter Bcl-2 expression of $(n=3, * * P>0.01)$. We obtained the same result with Western Blot (Figure. 5d-e). Thus, APOC1P1-3 may enhance the anoikis resistance by blocking the inhibition of miRNA-188-3p against the Bcl-2.

\section{Discussion}


Increasing reports indicate the importance of IncRNAs in the breast cancer metastasis [21, 22, 23], however, the relation between specific IncRNAs and anoikis resistance remains undetermined. In the current studies, we investigated the role of IncRNA APOC1P1-3 in anoikis resistance and its regulatory mechanisms. We found that it suppressed early apoptosis of breast cancer cells (MCF-7 and MDA-MB231) in the anoikis resistance model by activating Caspase 3, 8, 9 and PARP (Figure. 6). The miRNA-188$3 p$ is the potential target molecule (bioinformatics analysis and Dual-Luciferase report system). The IncRNA APOC1P1-3 suppresses miRNA-188-3p through competitively binding to alter the Bcl-2 expression.

LncRNAs play a crucial role in cancer development. Since anoikis resistance is critical for cancer metastases, IncRNAs could regulate the anoikis resistance to promote metastasis [24]. Indeed, Seitz et al. reported that IncRNA LINC00958 significantly reduced anoikis resistance in FL3 cell after knock-down [25]. We previously found that IncRNA APOC1P1-3 aberrantly overexpressed in breast cancer, and correlated with the tumor size and hypomethylation in its promoter. It decreased a-tubulin acetylation and apoptosis. In this study, we further established that it could promote anoikis resistance by regulating the early apoptosis.

Many proteins are involved in the apoptosis, and cause anoikis resistance. We investigated the relationship between IncRNA APOC1P1-3 and apoptotic proteins, and found that caspase 3, 8, 9 and PARP increased as the IncRNA APOC1P1-3 decreased. Whereas, activated caspase and PARP proteins decreased as the IncRNA APOC1P1-3 increased (Figure. 3e-f). All these results suggest that IncRNA APOC1P1-3 could promote anoikis resistance via caspase-dependent pathway. Indeed, activated-caspase proteins could promote apoptosis of cancer cells [26].

The IncRNAs could interplay with proteins, DNA, and RNA to regulate biological processes [27]. There is a cross-regulation between IncRNAs and microRNA $[28,29]$. LncRNAs interact with microRNA through different pathways. For example, micorRNA can influence the stability of IncRNAs. IncRNAs can act as miRNA decoys or sponges to repress target mRNAs [30]. LncRNAs can also compete with miRNAs by directly binding to the shared target mRNAs. In the current study, we isolated the IncRNA APOC1P1-3 from cytoplasm and explored its interaction with specific miRNAs. We found that miRNA-188-3p is most likely binding to IncRNA APOC1P1-3 (Figure. 4a) at 222-248 bp of APOC1P1-3 (Figure. 4b囚4d).

The miRNA-188-3p is a novel independent prognostic factor in colorectal cancer patients because it promotes cancer cell migration [31]. In addition, it negatively regulates NCAPG2 via activating NF-kb to block NCAPG2-mediated hepatocarcinoma proliferation and metastasis [32]. The miRNA-188-3p is also involved in sevoflurane-induced cognitive dysfunction via MDM2 proto-oncogene [33]. Furthermore, it negatively regulates TMED3 to inhibit the proliferation, migration and invasion of breast tumor [34]. These studies indicate that miRNA-188-3p has different functions in diverse pathological conditions. In our study, miRNA-188-3p promotes the early apoptosis of MDA-MB-231a cells, suggesting an inhibition of anoikis resistance. It specifically binds to IncRNA APOC1P1-3, and regulate the expression of Bcl-2 (Fig. 6) to promote the anoikis resistance. 


\section{Conclusion}

In summary, our study demonstrates that IncRNA APOC1P1-3 can promote the anoikis resistance of breast cancer cells and specifically bind to miRNA-188-3p acting as a "sponge" to block the inhibition of anti-apoptotic protein Bcl-2.

\section{Abbreviations}

IncRNA APOC1P1-3

long non-coding RNAs APOC1P1-3;

ECM

extracellular matrix;

ORF

Open Reading Frame;

polyHEMA

poly-2-hydroxycthyl methacrylate;

FACS

Fluorescence-activated cell sorting.

\section{Declarations}

\section{Ethics approval and consent to participate}

All studies involving breast cancer samples in our present research have been approved by Ethics Committee of Fudan University. All animal experiments were performed according to protocols approved by the Institutional Animal Care and Use Committee at Fudan University.

\section{Consent for publication}

All Authors have approved the manuscript and agree with submission to Journal of Experimental and Clinical Cancer Research.

\section{Availability of data materials}

The datasets generated and used in this study are available from the corresponding author on reasonable request.

\section{Competing interests}

The authors declare no conflict of interest.

\section{Funding}


This research was supported by grants from the Natural Science Foundation of China (81372849, 81874121); Natural Science Foundation of Shanghai (16ZR1403300).

\section{Authors' contributions}

Ping Zhou and Xingjun Qin conceived the project and supervised all studies. Qi Lu performed all experiments, analyzed and interpreted all the data. Li Wang constructed the manuscript and Jerry Yu refined and modified the article. Ping Zhu, Luying Li, Xue Wang and Youping Jin collected clinical sample and information. Xiuling Zhi and Xin Li support the experimental techniques. All authors participated in preparing the manuscript and approved the submitted and published version.

\section{Acknowledgements}

The authors wish to acknowledge Fudan University Shanghai Cancer Center and Shanghai Institute of Nutrition and Health, Chinese Academy of Sciences for providing the breast cancer tissue samples and luciferase detection equipments, respectively.

\section{References}

1. Cheung KJ, Ewald AJ. A collective route to metastasis: Seeding by tumor cell clusters. Science. 2016 Apr 8;352(6282):167-9.

2. Simpson CD, Anyiwe K, Schimmer AD. Anoikis resistance and tumor metastasis. Cancer Lett. 2008 Dec 18;272(2):177-85.

3. Paoli P, Giannoni E, Chiarugi P. Anoikis molecular pathways and its role in cancer progression. Biochim Biophys Acta. 2013 Dec;1833(12):3481-3498.

4. Kim YN, Koo KH, Sung JY, Yun UJ, Kim H. Anoikis resistance: an essential prerequisite for tumor metastasis. Int J Cell Biol. 2012; 2012:306879.

5. Wang KC, Chang HY. Molecular mechanisms of long noncoding RNAs. Mol Cell. 2011 Sep 16;43(6):904-14.

6. Ponting CP, Oliver PL, Reik W. Evolution and functions of long noncoding RNAs. Cell. 2009 Feb 20;136(4):629-41.

7. Batista PJ, Chang HY. Long noncoding RNAs: cellular address codes in development and disease. Cell. 2013 Mar 14;152(6):1298-307.

8. Kung JT, Colognori D, Lee JT. Long noncoding RNAs: past, present, and future. Genetics. 2013 Mar;193(3):651-69.

9. Huarte M. The emerging role of IncRNAs in cancer. Nat Med. 2015 Nov;21(11):1253-61.

10. Bhan A, Mandal SS. LncRNA HOTAIR: A master regulator of chromatin dynamics and cancer. Biochim Biophys Acta. 2015 Aug;1856(1):151-64.

11. Botti G, Scognamiglio G, Aquino G, Liguori G, Cantile M. LncRNA HOTAIR in Tumor Microenvironment: What Role? Int J Mol Sci. 2019 May 8;20(9). 
12. Wei MM, Zhou GB. Long Non-coding RNAs and Their Roles in Non-small-cell Lung Cancer. Genomics Proteomics Bioinformatics. 2016 Oct;14(5):280-288.

13. Xue D, Lu H, Xu HY, Zhou CX, He XZ. Long noncoding RNA MALAT1 enhances the docetaxel resistance of prostate cancer cells via miR-145-5p-mediated regulation of AKAP12. J Cell Mol Med. 2018 Jun;22(6):3223-3237.

14. Li P, Zhang X, Wang H, Wang L, Liu T, Du L, Yang Y, Wang C. MALAT1 Is Associated with Poor Response to Oxaliplatin-Based Chemotherapy in Colorectal Cancer Patients and Promotes Chemoresistance through EZH2. Mol Cancer Ther. 2017 Apr;16(4):739-751.

15. Xie H, Liao X, Chen Z, Fang Y, He A, Zhong Y, Gao Q, Xiao H, Li J, Huang W, Liu Y. LncRNA MALAT1 Inhibits Apoptosis and Promotes Invasion by Antagonizing miR-125b in Bladder Cancer Cells. J Cancer. 2017 Oct 17;8(18):3803-3811.

16. Crea F, Clermont PL, Parolia A, Wang Y, Helgason CD. The non-coding transcriptome as a dynamic regulator of cancer metastasis. Cancer Metastasis Rev. 2014 Mar;33(1):1-16.

17. Liao XH, Wang JG, Li LY, Zhou DM, Ren KH, Jin YT, Lv L, Yu JG, Yang JY, Lu Q, Zou Q, Yu J, Liu XP, Zhou P. Long intergenic non-coding RNA APOC1P1-3 inhibits apoptosis by decreasing a-tubulin acetylation in breast cancer. Cell Death Dis. 2016 May 26;7:e2236.

18. Lin MF, Jungreis I, Kellis M. PhyloCSF: A Comparative Genomics Method to Distinguish Protein Coding and Non-Coding Regions. Bioinformatics. 2011 Jul 1;27(13):i275-82.

19. Haemmerle M, Taylor ML, Gutschner T, Pradeep S, Cho MS, Sheng J, Lyons YM, Nagaraja AS, Dood RL, Wen Y, Mangala LS, Hansen JM, Rupaimoole R, Gharpure KM, Rodriguez-Aguayo C, Yim SY, Lee JS, Ivan C, Hu W, Lopez-Berestein G, Wong ST, Karlan BY, Levine DA, Liu J, Afshar-Kharghan V, Sood AK. Platelets reduce anoikis and promote metastasis by activating YAP1 signaling. Nat Commun. 2017 Aug 21;8(1):310.

20. Akekawatchai C, Roytrakul S, Kittisenachai S, Isarankura-Na-Ayudhya P, Jitrapakdee S. Protein Profiles Associated with Anoikis Resistance of Metastatic MDA-MB-231 Breast Cancer Cells. Asian Pac J Cancer Prev. 2016;17(2):581-90.

21. Soudyab M, Iranpour M, Ghafouri-Fard S. The Role of Long Non-Coding RNAs in Breast Cancer. Arch Iran Med. 2016 Jul;19(7):508-17.

22. Liu Y, Sharma S, Watabe K. Roles of IncRNA in breast cancer. Front Biosci (Schol Ed). 2015 Jun 1;7:94-108.

23. Bin X, Hongjian Y, Xiping Z, Bo C, Shifeng Y, Binbin T. Research progresses in roles of LncRNA and its relationships with breast cancer. Cancer Cell Int. 2018 Nov 12;18:179.

24. Kamarajugadda S, Cai Q, Chen H, Nayak S, Zhu J, He M, Jin Y, Zhang Y, Ai L, Martin SS, Tan M, Lu J. Manganese superoxide dismutase promotes anoikis resistance and tumor metastasis. Cell Death Dis. 2013 Feb 21;4:e504.

25. Seitz AK, Christensen LL, Christensen E, Faarkrog K, Ostenfeld MS, Hedegaard J, Nordentoft I, Nielsen MM, Palmfeldt J, Thomson M, Jensen MT, Nawroth R, Maurer T, Ørntoft TF, Jensen JB, Damgaard 
CK, Dyrskjøt L. Profiling of long non-coding RNAs identifies LINC00958 and LINC01296 as candidate oncogenes in bladder cancer. Sci Rep. 2017 Mar 24;7(1):395.

26. Budihardjo I, Oliver H, Lutter M, Luo X, Wang X. Biochemical pathways of caspase activation during apoptosis. Annu Rev Cell Dev Biol. 1999;15:269-90.

27. Quinn JJ, Chang HY. Unique features of long non-coding RNA biogenesis and function. Nat Rev Genet. 2016 Jan;17(1):47-62.

28. Marchese FP, Raimondi I, Huarte M. The multidimensional mechanisms of long noncoding RNA function. Genome Biol. 2017 Oct 31;18(1):206.

29. Ling H, Fabbri M, Calin GA. MicroRNAs and other non-coding RNAs as targets for anticancer drug development. Nat Rev Drug Discov. 2013 Nov;12(11):847-65.

30. Yoon JH, Abdelmohsen K, Gorospe M. Functional interactions among microRNAs and long noncoding RNAs. Semin Cell Dev Biol. 2014 Oct;34:9-14.

31. Salmena L, Poliseno L, Tay Y, Kats L, Pandolfi PP. A ceRNA hypothesis: the Rosetta Stone of a hidden RNA language? Cell. 2011 Aug 5;146(3):353-8.

32. Pichler M, Stiegelbauer V, Vychytilova-Faltejskova P, Ivan C, Ling H, Winter E, Zhang X, Goblirsch M, Wulf-Goldenberg A, Ohtsuka M, Haybaeck J, Svoboda M, Okugawa Y, Gerger A, Hoefler G, Goel A, Slaby 0, Calin GA. Genome-Wide miRNA Analysis Identifies miR-188-3p as a Novel Prognostic Marker and Molecular Factor Involved in Colorectal Carcinogenesis. Clin Cancer Res. 2017 Mar 1;23(5):13231333.

33. Meng F, Zhang S, Song R, Liu Y, Wang J, Liang Y, Wang J, Han J, Song X, Lu Z, Yang G, Pan S, Li X, Liu Y, Zhou F, Wang Y, Cui Y, Zhang B, Ma K, Zhang C, Sun Y, Xin M, Liu L. NCAPG2 overexpression promotes hepatocellular carcinoma proliferation and metastasis through activating the STAT3 and NF-kB/miR-188-3p pathways. EBioMedicine. 2019 Jun;44:237-249.

34. Wang L, Zheng M, Wu S, Niu Z. MicroRNA-188-3p is involved in sevoflurane anesthesia-induced neuroapoptosis by targeting MDM2. Mol Med Rep. 2018 Mar;17(3):4229-4236.

\section{Figures}


a
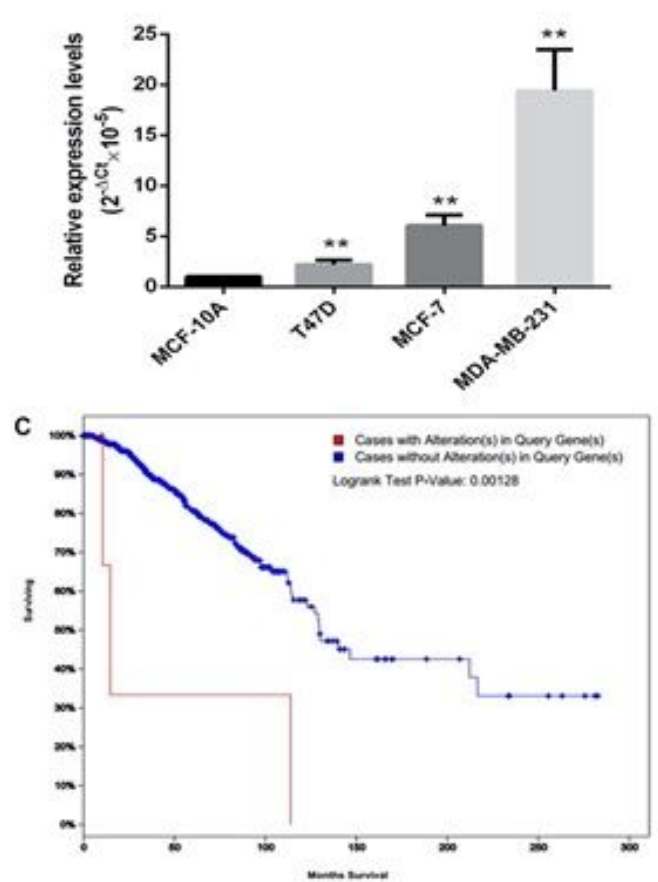

e i
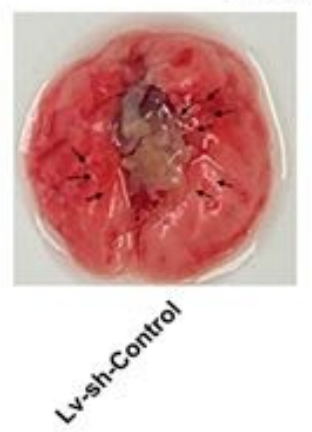

e ii

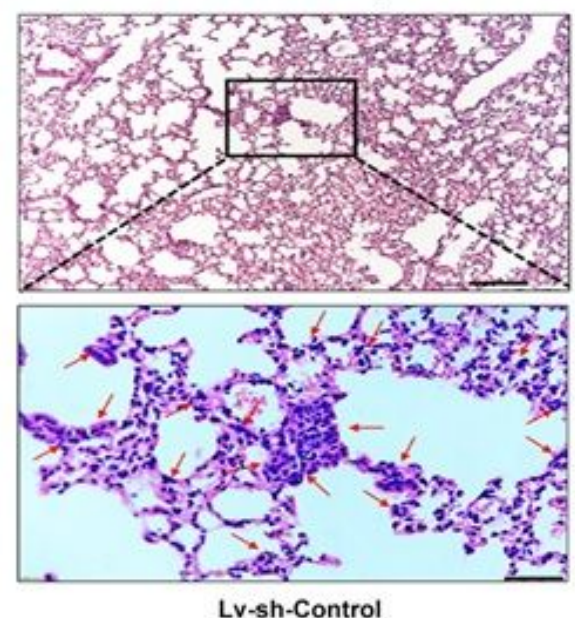

Lv-sh-Control b Nuclear fraction

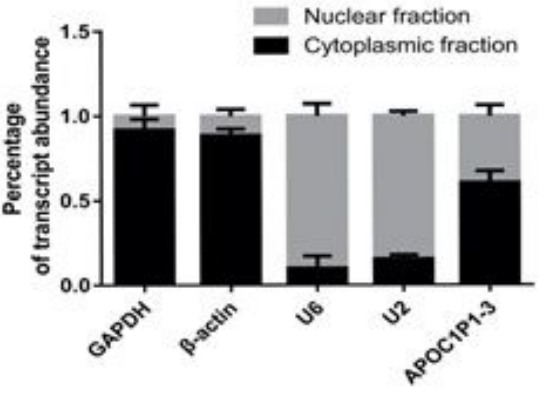

d
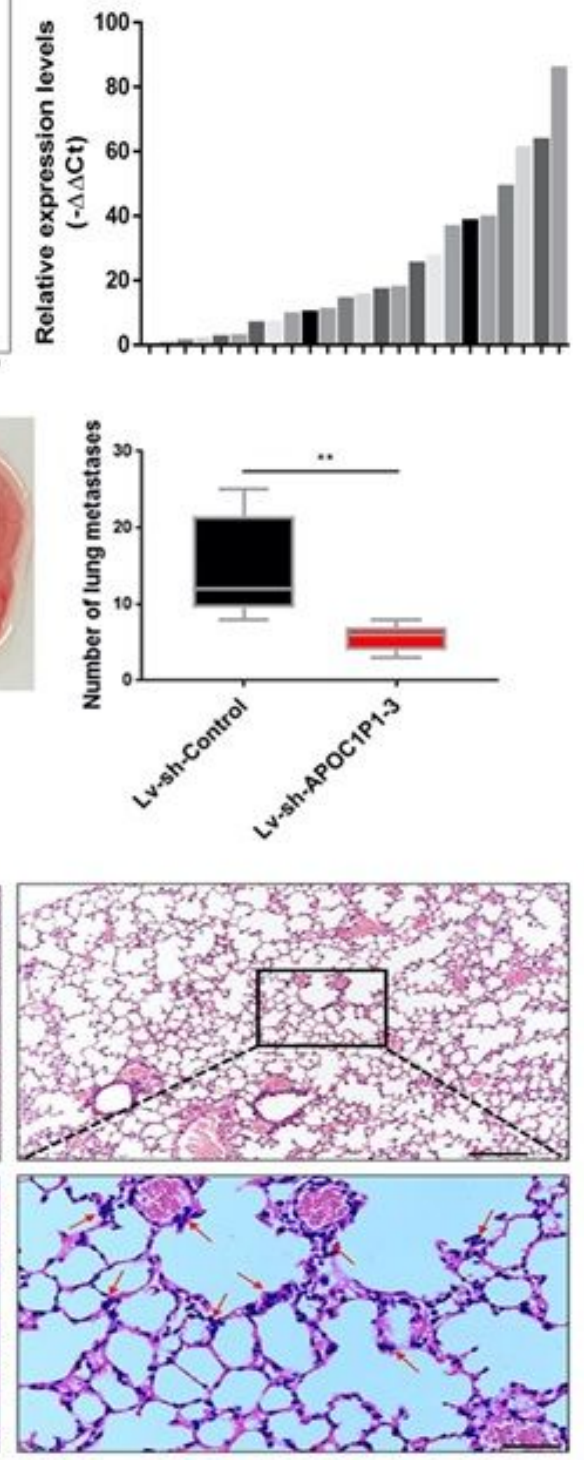

Lv-sh-APOC1P1-3

\section{Figure 1}

Correlation between IncRNA-APOC1P1-3 and breast cancer. a: The expression in breast cancer cells (T47D, MCF-7, MDA-MB-231) and normal mammary epithelial cell (MACF-10A) (RT-PCR). $n=3$, relative to the control group ${ }^{\star *} P<0.01$. b: In cytoplasmic nuclear separation of MDA-MB-231 cells, APOC1P1-3 expressed in different cell compartments (qRT-PCR). U6 RNA and U2 RNA were used for nuclear gene expression; and GAPDH RNA and $\beta$-catin RNA were used for cytoplasmic gene expression. c: The 
expression of IncRNA-APOC1P1-3 in 24 fresh breast cancer tissues (RT-PCR). d: The overall survival rate of breast cancer patients. Red line, high expression of APOC1P1-3 (110 months), blue line, low expression of APOC1P1-3 (300 months) ( $n=816, P<0.01)$. e: APOC1P1-3 promotes lung metastasis. (i) The number of lung metastases after tail vein injection with MDA-MB-231 cells infected with AP0C1P1-3-knock-down or control vector. (ii) Representative Hematoxylin and Eosin stain (H\&E) of lung metastases after the tail vein injection. Scale bars: two pictures above is $200 \mu \mathrm{m}$, two pictures below is $50 \mu \mathrm{m}$. ${ }^{\star *}, \mathrm{P}<0.01$.
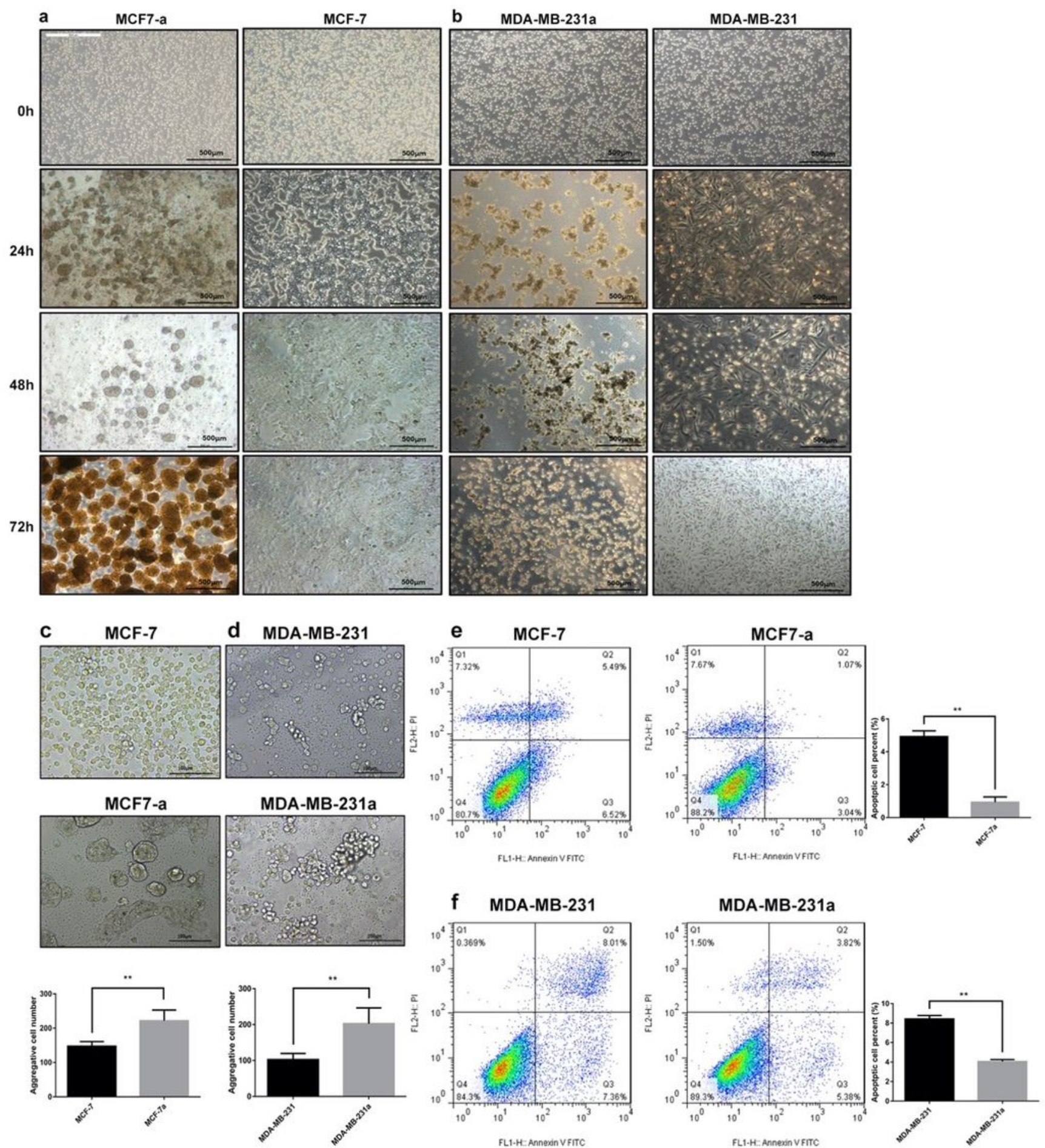

Figure 2 
Morphological changes, aggregation rate and apoptotic changes of anoikis resistant cells. a\&b: MCF7a/MDA-MB-231a cells were cultured in anoikis model for 24, 48 and 72h. MCF-7/MDA-MB-231 cells were cultured in adherent state as normal control. As the induction time of MCF-7a/MDA-MB-231a increases in the anoikis model, the suspended single breast cancer cells begin to aggregate gradually. c\&d: Microscopic morphology of MCF-7a/MDA-MB-231a and MCF-7/MDA-MB-231 cells $12 \mathrm{~h}$ culture in anoikis or normal groups. The aggregation rate in MCF-7a/MDA-MB-231a is significantly more than MCF7/MDA-MB-231 groups ( $n=7, * * P<0.01)$. e\&f: The effect of anoikis resistant on MCF-7a/ MDA-MB-231a cells (Annexin V-FITC/PI double staining). Early apoptosis was significantly reduced in the MCF-7a/MDAMB-231a group than in the MCF-7/MDA-MB-231 group $(n=3, * * P<0.01)$. 

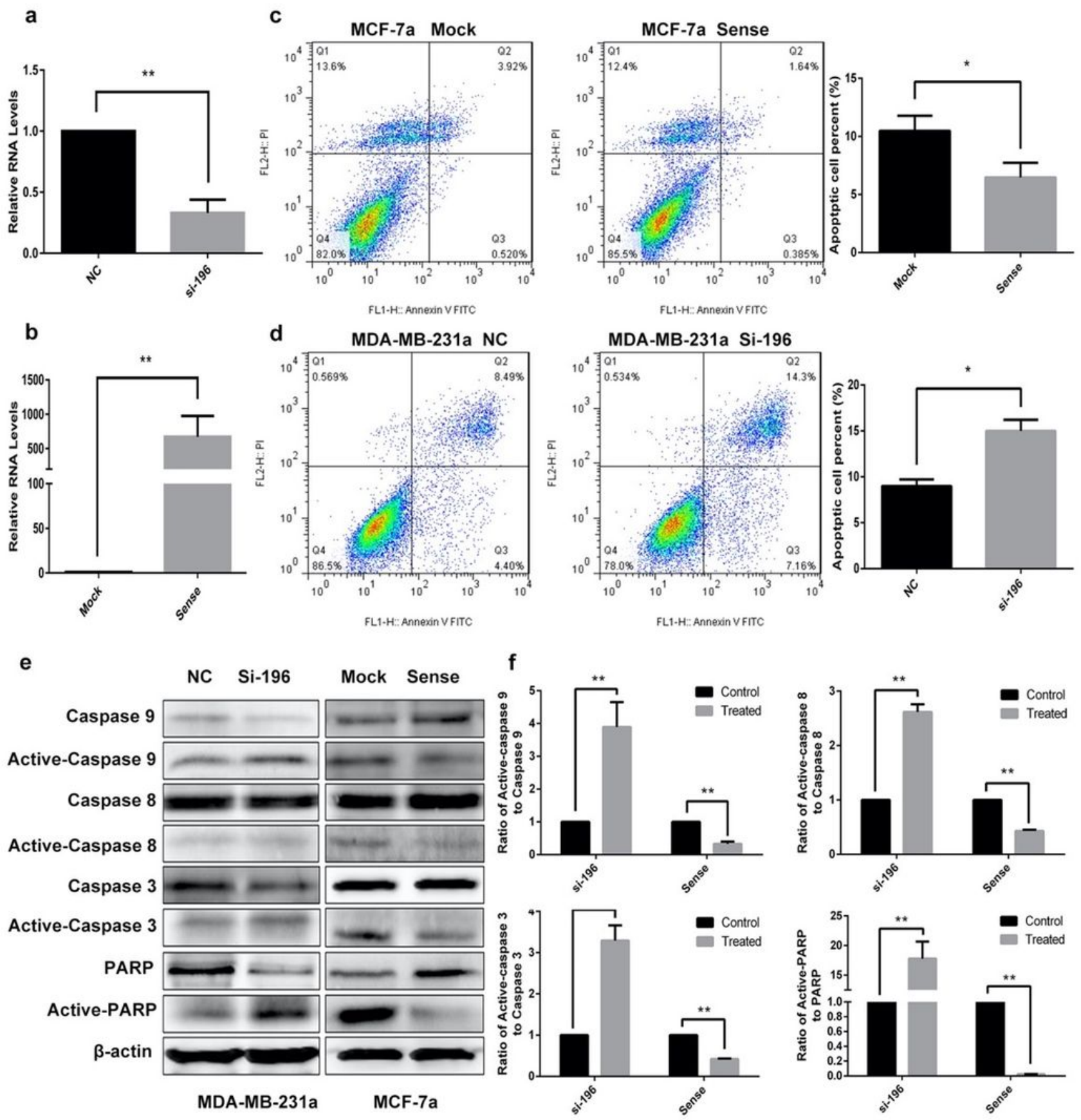

\section{Figure 3}

Effects of IncRNA-APOC1P1-3 on anoikis resistant. a\&b: The expression of APOC1P1-3 was inhibited in MDA-MB-231 cells transfected with siRNA-196, but increased in MCF-7 cells transfected with pcDNA3.1sense ( $q R T-P C R)\left(n=3{ }^{*} * * P<0.01\right)$. c\&d: Early apoptosis was more in MDA-MB-231a cells (low expression of APOC1P1-3) than in MCF-7a cells (high expression). e\&f: Apoptosis-related protein (Western Blot). Similarly, in MDA-MB-231a cells active-Caspase 3, 8,9 and active-PARP expressions and the shear rate of apoptotic proteins increased, but decreased in MCF-7a cells. 
a

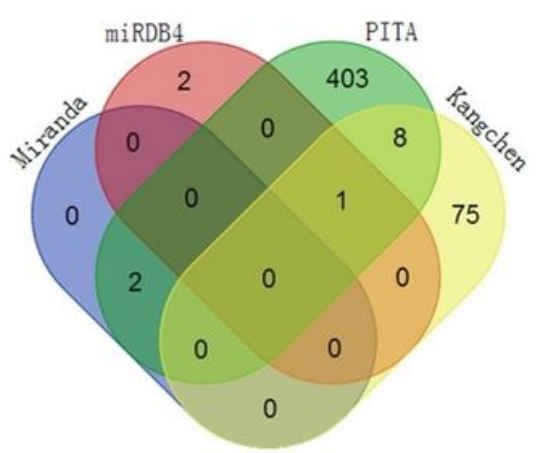

b
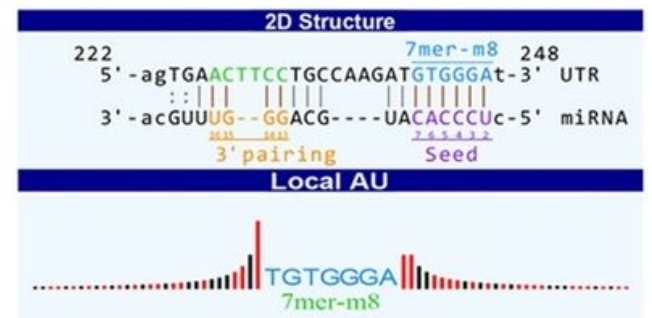

Position
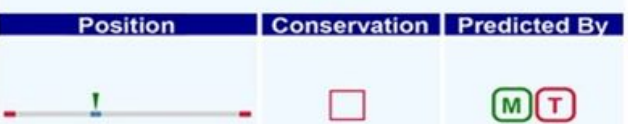

M $T$

c

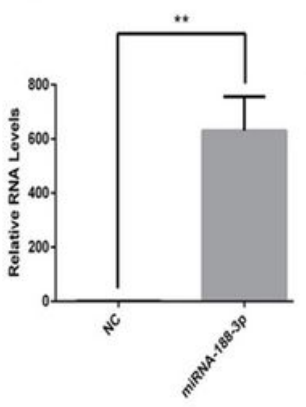

d

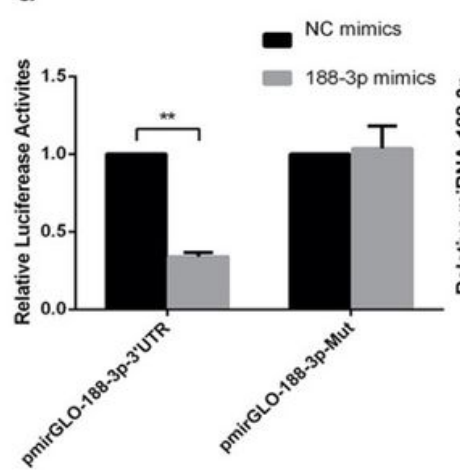

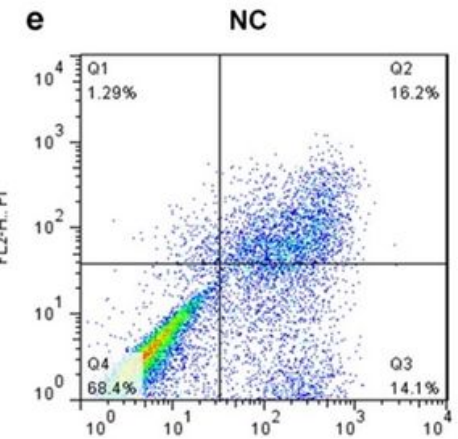

f

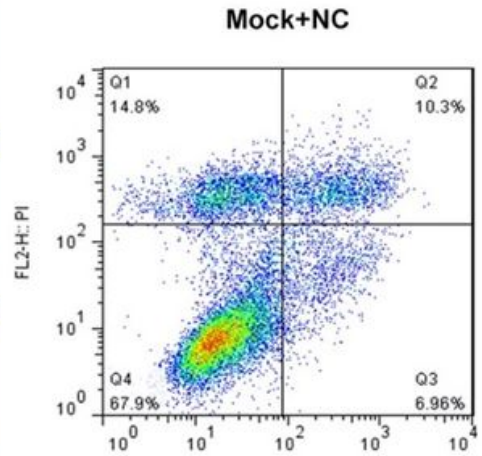

g

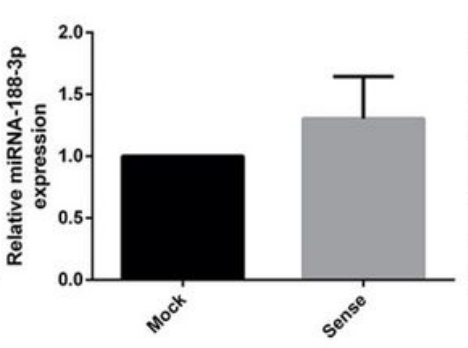

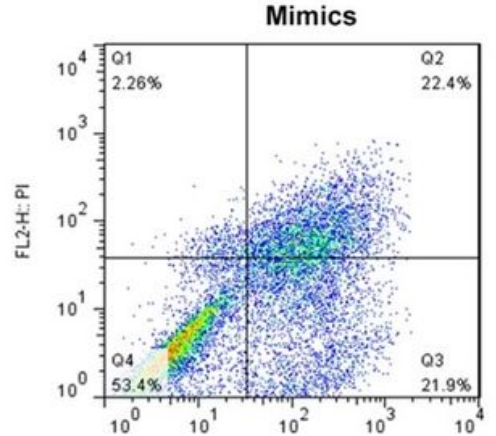

FL1-H: Annexin V FITC

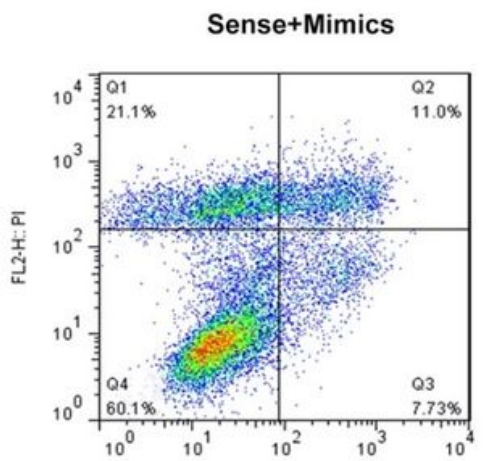

FL1-H: Annexin V FITC

h

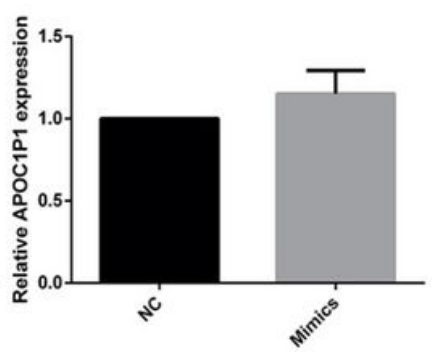

\section{Figure 4}

IncRNA-APOC1P1-3 binds to miRNA-188-3p, and miRNA-188-3p reverses the anti-apoptotic effect of LincRNA-AP0C1P1-3. a: Venn diagram of target microRNAs predicted by multiple databases. b: RNA-chip predicted binding sites of APOC1P1-3 with miRNA-188-3p. c: The overexpression of transfected miRNA 188-3p mimics was detected by qPCR, which was significantly higher than that in the control group $(n=3$, $\left.{ }^{*} \mathrm{P}<0.01\right)$.d: Combination of APOC1P1-3 with miRNA-188-3P. When the pmirGLO-APOC1P1-3'UTR plasmid and miRNA-188-3P mimics were co-transfected, the fluorescent activity significantly decreased. When the pmirGLO-APOC1P1-Mut plasmid and miRNA-188-3P mimics were co-transfected, the fluorescent activity was restored. e: Compared with the control group, early apoptosis of MDA-MB-231a cells transfected with miRNA-188-3p mimics increased significantly (FACS). f: Overexpression of miRNA188-3p followed by overexpression of IncRNA-APC1P1-3 reversed its pro-apoptotic effect(FACS). g\&h: 
There's no difference in the expression level of IncRNA-APC1P1-3 after overexpressing miRNA-188-3p (qRT-PCR). After overexpressing IncRNA-APC1P1-3, the expression of miRNA-188-3p was also unchanged.

a

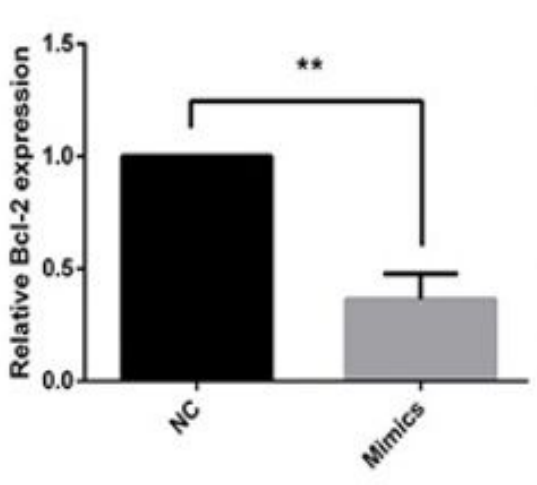

d b

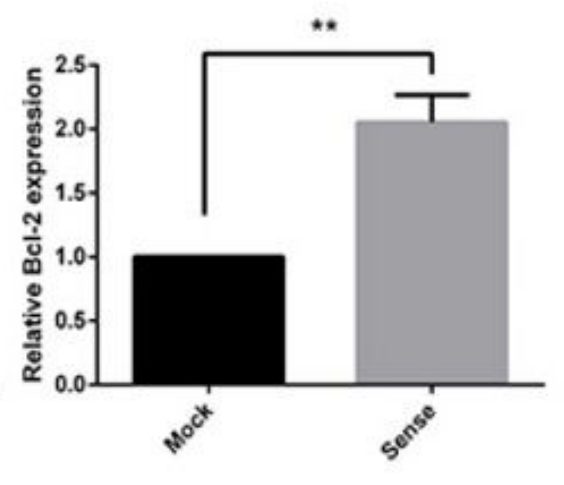

C

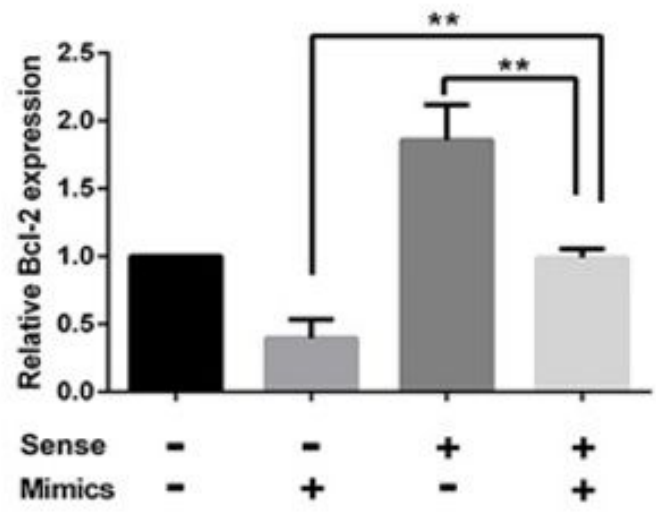

e
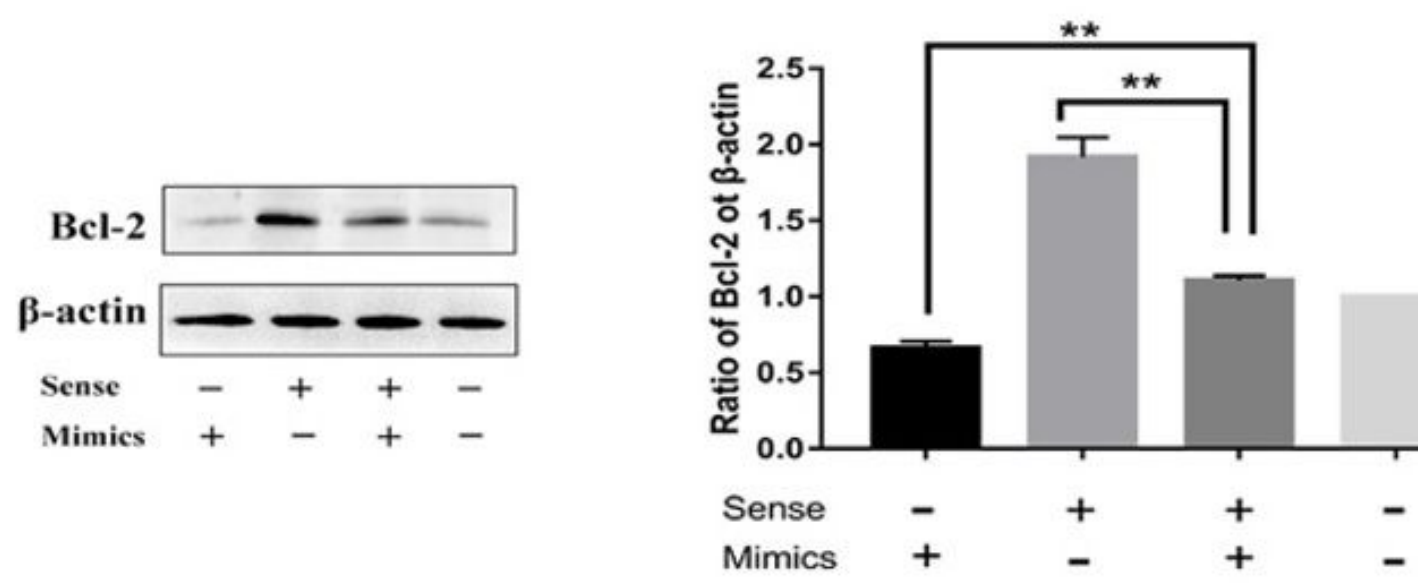

Figure 5

Effects of LncRNA-APC1P1-3 and miRNA-188-3p on the expression of Bcl-2. a\&b: Overexpression of miRNA-188-3p decreases, but overexpression of IncRNA-APC1P1-3 increases the expression of Bcl-2. c: Overexpression of IncRNA-APC1P1-3 followed by up-regulation of miRNA-188-3p abolished the IncRNAAPC1P1-3 effect on Bcl-2. d\&e: In four groups (sense+/mimics-, sense-/mimicst, sense+/mimicst, sense-/mimics-) simultaneous transfection of sense and mimics reversed the expression of Bcl-2 when separately transfected with sense/mimics. 


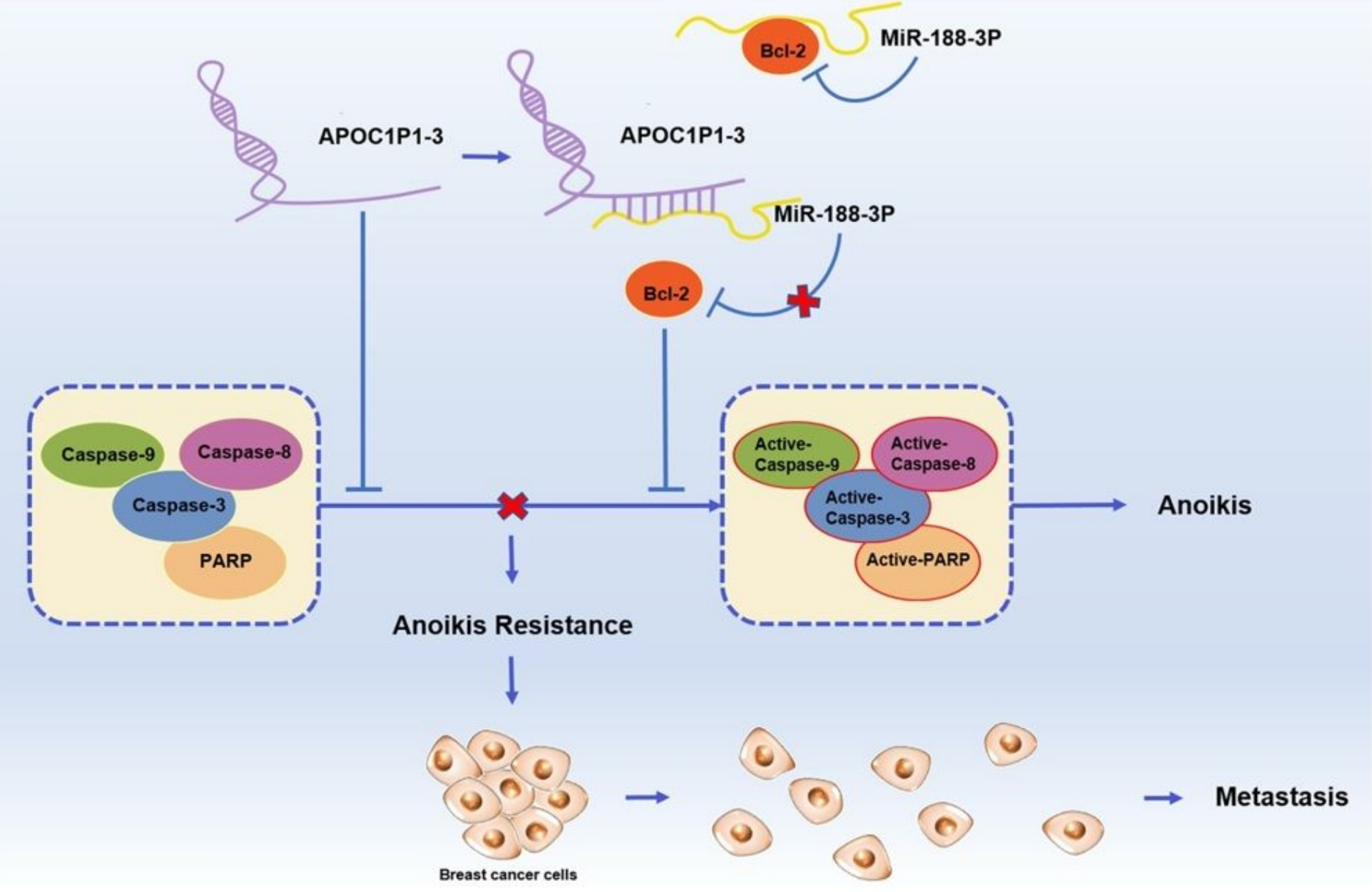

Figure 6

The molecular mechanism of LncRNA-APC1P1-3 in anoikis resistance. Two pathways, IncRNA APOC1P13 activates Caspase 3, 8, 9 and PARP, and specifically binds to miRNA-188-3p to block its inhibition of Bcl2.

\section{Supplementary Files}

This is a list of supplementary files associated with this preprint. Click to download.

- Additionalfile1.pdf 